\title{
ON BOUNDED PSEUDODIFFERENTIAL OPERATORS IN A HIGH-DIMENSIONAL SETTING
}

\author{
L. AMOUR, L. JAGER, AND J. NOURRIGAT \\ (Communicated by Michael Hitrik) \\ Dedicated to the memory of Bernard Lascar
}

\begin{abstract}
This work is concerned with extending the results of Calderón and Vaillancourt, proving the boundedness of Weyl pseudodifferential operators $O p_{h}^{W e y l}(F)$ in $L^{2}\left(\mathbb{R}^{n}\right)$. We state conditions under which the norm of such operators has an upper bound independent of $n$. To this aim, we apply a decomposition of the identity to the symbol $F$, thus obtaining a sum of operators of a hybrid type, each of them behaving as a Weyl operator with respect to some of the variables and as an anti-Wick operator with respect to the other ones. Then we establish upper bounds for these auxiliary operators, using suitably adapted classical methods like coherent states.
\end{abstract}

\section{INTRODUCTION}

Since the work of Calderón and Vaillancourt [2], it is well known that if a function $F$, defined on $\mathbb{R}^{2 n}$, is smooth and has bounded derivatives, it is possible to associate with it a pseudodifferential operator, depending on a parameter $h>0$, which is bounded on $L^{2}\left(\mathbb{R}^{n}\right)$ (see also $\left.7,19,20,22\right]$ ). This operator is formally defined by $(1.1)$

$$
\left(O p_{h}^{W e y l}(F) f\right)(x)=(2 \pi h)^{-n} \int_{\mathbb{R}^{2 n}} e^{\frac{i}{h}(x-y) \cdot \xi} F\left(\frac{x+y}{2}, \xi\right) f(y) d y d \xi, \quad x \in \mathbb{R}^{n},
$$

for $f$ belonging to $L^{2}\left(\mathbb{R}^{n}\right)$. (When $h=1$ the subscript $h$ will be omitted.) Moreover, its norm is bounded above by

$$
\left\|O p_{h}^{W e y l}(F)\right\|_{\mathcal{L}\left(L^{2}\left(\mathbb{R}^{n}\right)\right)} \leq C \sum_{|\alpha+\beta| \leq N}\left\|\partial_{x}^{\alpha} \partial_{\xi}^{\beta} F\right\|_{L^{\infty}\left(\mathbb{R}^{2 n}\right)},
$$

where $N$ and $C$ depend on the dimension $n$.

The aim of this work is to prove that, under certain conditions, the constants appearing in the upper bound do not depend on the dimension. The set of derivation multi-indices which are used depends on the dimension in a way that will be precisely stated.

We shall thus be able to give examples where the dimension goes to infinity and the norm, nevertheless, remains bounded.

In a later work we shall study pseudodifferential operators where the configuration space $\mathbb{R}^{n}$ will be replaced by an infinite dimensional Hilbert space, by a method

Received by the editors March 11, 2013 and, in revised form, July 13, 2013, September 6, 2013, September 19, 2013, and October 9, 2013.

2010 Mathematics Subject Classification. Primary 35S05. 
differing from Bernard Lascar's (see [9]-[18). These results have been announced in a preprint [1] in September 2012.

We first recall an example in which the constant appearing in the upper bound on the norm does not depend on the dimension. This is the case when the function $F$ is the Fourier transform of a function $G$ belonging to $L^{1}\left(\mathbb{R}^{n}\right)$ :

$$
F(x, \xi)=(2 \pi h)^{-2 n} \int_{\mathbb{R}^{2 n}} e^{\frac{i}{h}(a \cdot x+b \cdot \xi)} G(a, b) d a d b .
$$

Since the Weyl operator associated with the function

$$
(x, \xi) \rightarrow E_{a, b, h}(x, \xi)=e^{\frac{i}{h}(a \cdot x+b \cdot \xi)}
$$

is the operator $W_{a, b, h}$ defined by

$$
\left(O p_{h}^{W e y l}\left(E_{a, b, h}\right) f\right)(u)=\left(W_{a, b, h} f\right)(u)=e^{\frac{i}{h} a \cdot u+\frac{i}{2 h} a \cdot b} f(u+b),
$$

the equality (1.1) may be rewritten in the form

$$
O p_{h}^{W e y l}(F)=(2 \pi h)^{-2 n} \int_{\mathbb{R}^{2 n}} G(a, b) W_{a, b, h} d a d b .
$$

Since $W_{a, b, h}$ is unitary,

$$
\left\|O p_{h}^{W e y l}(F)\right\| \leq(2 \pi h)^{-2 n} \int_{\mathbb{R}^{2 n}}|G(a, b)| d a d b .
$$

Situations of this kind have been considered by B. Lascar ([9]-[18]) in an infinite dimensional setting, but the $L^{2}$ boundedness was not the main motivation of these works.

Our approach is different, in that we aim at extending the bound (1.2). Let us specify the set of multi-indices which will be used. Cordes [5], Coifman Meyer [3], and Hwang 8 noticed that one does not need all the multi-indices to state (1.2), but only the $(\alpha, \beta)$ satisfying $0 \leq \alpha_{j} \leq 1$ and $0 \leq \beta_{j} \leq 1$ for each $j$. In this paper we shall use the multi-indices $(\alpha, \beta)$ such that $0 \leq \alpha_{j} \leq 2$ and $0 \leq \beta_{j} \leq 2$ for each $j$. We now can state the hypotheses on the function $F$.

Let $\left(\rho_{j}\right)_{1 \leq j \leq n}$ and $\left(\delta_{j}\right)_{1 \leq j \leq n}$ be two sequences satisfying $\rho_{j} \geq 0$ and $\delta_{j} \geq 0$ for every $j \leq n$, and let $M$ be a nonnegative real number. Suppose that

$(\mathrm{H})$ for every multi-index $(\alpha, \beta)$ such that $0 \leq \alpha_{j} \leq 2$ and $0 \leq \beta_{j} \leq 2$ for every $j \leq n$, the partial derivative $\partial_{x}^{\alpha} \partial_{\xi}^{\beta} F$ exists, is continuous, bounded and satisfies

$$
\left|\partial_{x}^{\alpha} \partial_{\xi}^{\beta} F(x, \xi)\right| \leq M \prod_{j=1}^{n} \rho_{j}^{\alpha_{j}} \delta_{j}^{\beta_{j}} .
$$

If $\rho_{j}=0$ and $\alpha_{j}=0$, we set that $\rho_{j}^{\alpha_{j}}=1$.

Our main result is the following theorem.

Theorem 1.1. If a function $F$ defined on $\mathbb{R}^{2 n}$ satisfies hypothesis $(H)$, then the operator $O p_{h}^{W e y l}(F)$, defined formally by (1.1), is bounded in $L^{2}\left(\mathbb{R}^{n}\right)$ and satisfies

$$
\left\|O p_{h}^{W e y l}(F)\right\|_{\mathcal{L}\left(L^{2}\left(\mathbb{R}^{n}\right)\right)} \leq M \prod_{j=1}^{n}\left(1+81 \pi h \rho_{j} \delta_{j}\right)
$$

if $0<h \rho_{j} \delta_{j} \leq 1$ for every $j \leq n$. 
Example 1.2. Let $V \geq 0$ be a real-valued bounded function in $C^{\infty}(\mathbb{R})$, whose derivatives are all bounded. For all integer $n \geq 1$, set

$$
H_{n}(x, \xi)=\sum_{j \leq n} \xi_{j}^{2}+\sum_{\substack{j \leq n, k \leq n \\|j-k|=1}} g_{j} g_{k} V\left(x_{j}-x_{k}\right),
$$

where $\left(g_{j}\right)$ is a sequence of positive numbers such that, for some $C_{0}>0$, we have $g_{j} \leq C_{0} g_{k}$ if $|j-k| \leq 1$. Set

$$
P_{n}(x, \xi)=e^{-H_{n}(x, \xi)} .
$$

We shall see that hypothesis $(\mathrm{H})$ is satisfied, with

$$
M=1,
$$$$
\delta_{j}=C_{1},
$$$$
\rho_{j}=C_{1} \lambda_{j}
$$

$$
\lambda_{j}=\max _{1 \leq \nu \leq 4}\left(g_{j}^{2}\left\|V^{(\nu)}\right\|_{L^{\infty}}\right)^{1 / \nu},
$$

where $C_{1}$ is a real constant, to be determined, depending only on $C_{0}$. Let us set

$$
W(x)=-\sum_{\substack{j \leq n, k \leq n \\|j-k|=1}} g_{j} g_{k} V\left(x_{j}-x_{k}\right)
$$

and

$$
A_{n}=\{(j, k),|j-k|=1, \quad 1 \leq j, k \leq n\} .
$$

We shall estimate $\partial^{\alpha} e^{W}$ when $\alpha_{j} \leq 2$ for every $j$. For each function $f \in C^{4}(\mathbb{R})$ with bounded derivatives, set

$$
M(f)=\max _{1 \leq \nu \leq 4}\left(\left\|f^{(\nu)}\right\|_{L^{\infty}}\right)^{1 / \nu} .
$$

We notice that

$$
e^{-f} \partial^{\nu} e^{f}=\left(\partial+f^{\prime}\right)^{\nu} \cdot 1
$$

From this and a simple computation follows that

$$
\left|e^{-f} \partial^{\nu} e^{f}\right| \leq(2 M(f))^{\nu} .
$$

In order to apply this inequality we divide $W$ into two parts. Set

$$
U_{j}=U_{j}\left(x_{1}, \ldots, x_{n}\right)=-g_{j} g_{j+1} U\left(x_{j}-x_{j+1}\right), \quad U(y)=V(y)+V(-y)
$$

and

$$
W_{e}=\sum_{j \text { even }} U_{j}, \quad W_{o}=\sum_{j \text { odd }} U_{j}
$$

Then $W=W_{e}+W_{o}$, and we notice that the variable $x_{\nu}$ occurs only once in the $W_{e}$ and $W_{o}$. Also, since

$$
\partial^{\alpha} e^{W_{e}}=\prod_{j \text { even, } j<n}\left(\partial_{j}^{\alpha_{j}} \partial_{j+1}^{\alpha_{j+1}} e^{U_{j}}\right)
$$

we have the estimate

$$
\left|\partial^{\alpha} e^{W_{e}}\right| \leq e^{W_{e}} \prod_{j \text { even, } j<n} T_{j},
$$

where $T_{j}$ is the $L^{\infty}$ norm of the function

$$
e^{f_{j}} \partial^{\alpha_{j}+\alpha_{j+1}} e^{-f_{j}}, \quad f_{j}=g_{j} g_{j+1} U(x) .
$$

Let $\lambda_{j}$ be defined by (1.7), and set

$$
M_{j}=\max _{1 \leq \nu \leq 4}\left(\left\|g_{j} g_{j+1} U^{(\nu)}\right\|_{L^{\infty}}\right)^{1 / \nu} .
$$


Then

$$
M_{j} \leq 2 C_{0} \lambda_{j}, \quad M_{j} \leq 2 C_{0} \lambda_{j+1},
$$

where $C_{0}$ is defined so that $g_{j} g_{j+1} \leq C_{0} \min \left(g_{j}^{2}, g_{j+1}^{2}\right)$. It follows from (1.8) and (1.10) that

$$
T_{j} \leq\left(2 M_{j}\right)^{\alpha_{j}+\alpha_{j+1}} \leq\left(4 C_{0} \lambda_{j}\right)^{\alpha_{j}}\left(4 C_{0} \lambda_{j+1}\right)^{\alpha_{j+1}} .
$$

Then (1.9) gives

$$
\left|\partial^{\alpha} e^{W_{e}}\right| \leq e^{W_{e}} \prod_{j \text { even }, j<n}\left(4 C_{0} \lambda_{j}\right)^{\alpha_{j}}\left(4 C_{0} \lambda_{j+1}\right)^{\alpha_{j+1}} .
$$

In a similar way one gets the estimate

$$
\left|\partial^{\alpha} e^{W_{o}}\right| \leq e^{W_{o}} \prod_{j \text { odd }, j<n}\left(4 C_{0} \lambda_{j}\right)^{\alpha_{j}}\left(4 C_{0} \lambda_{j+1}\right)^{\alpha_{j+1}}
$$

Then we write

$$
\partial^{\alpha} e^{W}=\partial^{\alpha} e^{W_{e}} e^{W_{o}}=\sum^{\prime}\left(\begin{array}{l}
\alpha \\
\beta
\end{array}\right)\left(\partial^{\alpha-\beta} e^{W_{e}}\right)\left(\partial^{\beta} e^{W_{o}}\right),
$$

where the prime indicates that one only takes the summation over terms with $\beta_{1}=\alpha_{1}$ and $\beta_{n}=\alpha_{n}$ if $n$ is even, and with $\beta_{1}=\alpha_{1}$ and $\beta_{n}=0$ if $n$ is odd. We get the estimate

$$
\begin{gathered}
\left|\partial^{\alpha} e^{W}\right| \leq e^{W} \sum^{\prime}\left(\begin{array}{l}
\alpha \\
\beta
\end{array}\right)\left(\prod_{j \text { even, }, j<n}\left(4 C_{0} \lambda_{j}\right)^{\alpha_{j}-\beta_{j}}\left(4 C_{0} \lambda_{j+1}\right)^{\alpha_{j+1}-\beta_{j+1}}\right) \\
\cdot\left(\prod_{j \text { odd, } j<n}\left(4 C_{0} \lambda_{j}\right)^{\beta_{j}}\left(4 C_{0} \lambda_{j+1}\right)^{\beta_{j+1}}\right) \\
=e^{W} \sum^{\prime}\left(\begin{array}{l}
\alpha \\
\beta
\end{array}\right) \prod_{j=1}^{n}\left(4 C_{0} \lambda_{j}\right)^{\alpha_{j}} \leq 2^{|\alpha|} e^{W} \prod_{j=1}^{n}\left(4 C_{0} \lambda_{j}\right)^{\alpha_{j}} .
\end{gathered}
$$

We have proved that

$$
\left|\partial^{\alpha} e^{W}\right| \leq e^{W} \prod_{j=1}^{n}\left(8 C_{0} \lambda_{j}\right)^{\alpha_{j}}
$$

Therefore, hypothesis $(\mathrm{H})$ is satisfied with the choice of (1.6). We may apply Theorem 1.1 if $h \rho_{j} \delta_{j} \leq 1$ for all $j$. It follows that $\left\|O p_{h}^{W e y l}\left(P_{n}\right)\right\|_{\mathcal{L}\left(L^{2}\left(\mathbb{R}^{n}\right)\right)}$ is bounded independently of $n$ if the sum $\sum_{j \geq 1} g_{j}^{1 / 2}$ converges and if $h$ is small enough. If $g_{j}=1$, the norm is not bounded, but estimated, with some constant $C>0$, independent of the dimension, by

$$
\left\|O p_{h}^{W e y l}\left(P_{n}\right)\right\|_{\mathcal{L}\left(L^{2}\left(\mathbb{R}^{n}\right)\right)} \leq e^{C h n} .
$$

Example 1.3. The mean-field approximation uses hamiltonians of the form

$$
H_{n}(x, \xi)=\sum_{j \leq n} \xi_{j}^{2}+\frac{1}{n} \sum_{j \leq n, k \leq n} V\left(x_{j}-x_{k}\right),
$$

where $V$ is as in Example 1.2. Let $P_{n}$ be the function defined as in (1.5). Then hypothesis $(\mathrm{H})$ is satisfied with $M=1$ and $\rho_{j}=\delta_{j}=C_{1}$, where $C_{1}$ does not depend on $n$. In this case, Theorem 1.1 shows that, provided $C_{1}$ is small enough, we also have (1.11) for some constant $C$ which is independent of $n$. 


\section{Hybrid Weyl anti-Wick quantization}

In order to prove Theorem 1.1, we may as well assume that $\rho_{j}=\delta_{j}$ for every $j \leq n$ and that $h=1$. Indeed, if a function $F$ satisfies hypothesis $(\mathrm{H})$ with two sequences $\left(\rho_{j}\right)$ and $\left(\delta_{j}\right)$ of positive real numbers, then the function $\widetilde{F}$ defined by

$$
\widetilde{F}(x, \xi)=F\left(x_{1} \sqrt{h} \lambda_{1}, \ldots, x_{n} \sqrt{h} \lambda_{n}, \frac{\xi_{1} \sqrt{h}}{\lambda_{1}}, \ldots, \frac{\xi_{n} \sqrt{h}}{\lambda_{n}}\right), \quad \lambda_{j}=\sqrt{\frac{\delta_{j}}{\rho_{j}}},
$$

satisfies $(\mathrm{H})$ with $\rho_{j}$ and $\delta_{j}$ replaced by $\varepsilon_{j}=\sqrt{h \rho_{j} \delta_{j}}$. If Theorem 1.1 is valid for $O p_{1}^{W e y l}$, then we get that, if $\varepsilon_{j}^{2} \leq 1$,

$$
\left\|O p_{1}^{W e y l}(\widetilde{F})\right\|_{\mathcal{L}\left(L^{2}\left(\mathbb{R}^{n}\right)\right)} \leq M \prod_{j=1}^{n}\left(1+81 \pi \varepsilon_{j}^{2}\right) .
$$

Since $O p_{h}^{W e y l}(F)=T^{-1} O p_{1}^{W e y l}(\widetilde{F}) T$, where $T$ is a unitary operator acting in $L^{2}\left(\mathbb{R}^{n}\right)$, Theorem 1.1 for $O p_{h}^{W e y l}(F)$ holds true. This follows by continuity if some of the $\rho_{j}$ or $\delta_{j}$ are equal to 0 .

Consequently, we shall assume from now on that $\rho_{j}=\delta_{j} \leq 1$ for every $j \leq n$ and that $h=1$.

In order to prove Theorem 1.1 we shall split the operator into a sum of operators which will behave as Weyl operators with respect to a first subset of the variables (meaning the operators will be defined by a formula analogous to (1.1) in which only these variables appear) and as anti-Wick operators with respect to the other variables.

We first need to recall the anti-Wick quantization. The definition uses the coherent states, which are the family of functions $\Psi_{X}$ indexed by $X=(x, \xi) \in \mathbb{R}^{2 n}$, defined by

$$
\Psi_{X}(u)=\pi^{-n / 4} e^{-\frac{|u-x|^{2}}{2}} e^{i u . \xi-\frac{i}{2} x . \xi}, \quad X=(x, \xi) \in \mathbb{R}^{2 n}, \quad u \in \mathbb{R}^{n} .
$$

Recall that

$$
\langle f, g\rangle=(2 \pi)^{-n} \int_{\mathbb{R}^{2 n}}\left\langle f, \Psi_{X}\right\rangle\left\langle\Psi_{X}, g\right\rangle d X .
$$

If $F$ is a function in $L^{\infty}\left(\mathbb{R}^{2 n}\right)$, one can associate with it an (anti-Wick) operator $O p^{A W}(F)$ such that, for all $f$ and $g$ in $L^{2}\left(\mathbb{R}^{n}\right)$,

$$
\left\langle O p^{A W}(F) f, g\right\rangle=(2 \pi)^{-n} \int_{\mathbb{R}^{2 n}} F(X)\left\langle f, \Psi_{X}\right\rangle\left\langle\Psi_{X}, g\right\rangle d X .
$$

We then have

$$
\left\|O p^{A W}(F)\right\|_{\mathcal{L}\left(L^{2}\left(\mathbb{R}^{n}\right)\right)} \leq\|F\|_{L^{\infty}\left(\mathbb{R}^{2 n}\right)} .
$$

The relationship between Weyl and anti-Wick quantizations is given, for every $F$ in $L^{\infty}\left(\mathbb{R}^{2 n}\right)$, by

$$
O p^{A W}(F)=O p^{W e y l}\left(e^{\frac{1}{4} \Delta} F\right)
$$

where

$$
\Delta=\sum_{j \leq n} \Delta_{j}, \quad \Delta_{j}=\frac{\partial^{2}}{\partial x_{j}^{2}}+\frac{\partial^{2}}{\partial \xi_{j}^{2}}
$$


This fact is classical (see Folland [6]). One has an identity decomposition in $L^{\infty}\left(\mathbb{R}^{2 n}\right)$ :

$$
\begin{aligned}
I= & \sum_{E \subseteq\{1, \ldots, n\}} T(E) e^{\frac{1}{4} \Delta_{E^{c}}}, \\
\Delta_{E^{c}} & =\sum_{j \in E^{c}} \Delta_{j} .
\end{aligned}
$$

For every subset $E \subseteq\{1, \ldots, n\}$ and every symbol $F$, we define an operator $O p^{h y b, E}(F)$ by

$$
O p^{h y b, E}(F)=O p^{W e y l}\left(e^{\frac{1}{4} \Delta_{E^{c}}} F\right) .
$$

This operator behaves as a Weyl operator with respect to the variables $x_{j}(j \in E)$ and as an anti-Wick operator with respect to the variables $x_{j}\left(j \in E^{c}\right)$. If $E=\emptyset$, it is the anti-Wick operator, and conversely if $E=\{1, \ldots, n\}$, it is the Weyl operator.

One derives a decomposition of the Weyl operator $O p^{W e y l}(F)$ :

$$
O p^{W e y l}(F)=\sum_{E \subseteq\{1, \ldots, n\}} O p^{h y b, E}(T(E) F) .
$$

We shall now prove an upper bound on the norm of a hybrid operator $O p^{h y b, E}(G)$, where the function $G$ is bounded on $\mathbb{R}^{2 n}$. The only derivatives of $G$ which will play a role are the derivatives with respect to $x_{j}$ or $\xi_{j}$ with $j \in E$. For every integer $m$ we introduce the set of multi-indices

$I_{m}(E)=\left\{(\alpha, \beta), \quad \alpha_{j} \leq m, \quad \beta_{j} \leq m, \quad(1 \leq j \leq n) \quad \alpha_{j}=\beta_{j}=0 \quad\right.$ if $\left.j \notin E\right\}$.

We shall prove the following Lemma in Section 3, by adapting classical methods (Unterberger [22]).

Lemma 2.1. If $F$ satisfies hypothesis (H) and if $E \neq \emptyset$, then

$$
\left\|O p^{h y b, E}(F)\right\|_{\mathcal{L}\left(L^{2}\left(\mathbb{R}^{n}\right)\right)} \leq\left(\frac{9 \pi}{2}\right)^{|E|} \sum_{(\alpha, \beta) \in I_{2}(E)}\left\|\partial_{x}^{\alpha} \partial_{\xi}^{\beta} F\right\|_{L^{\infty}\left(\mathbb{R}^{2 n}\right)} .
$$

We shall establish the following lemma in Section 4.

Lemma 2.2. If $F$ satisfies hypothesis $(H)$ with $\rho_{j}=\delta_{j} \leq 1$ for every $j \leq n$, and if $E \neq \emptyset$, the function $T(E) F$ satisfies

$$
\sum_{(\alpha, \beta) \in I_{2}(E)}\left\|\partial_{x}^{\alpha} \partial_{\xi}^{\beta} T(E) F\right\|_{L^{\infty}\left(\mathbb{R}^{2 n}\right)} \leq M 18^{|E|} \prod_{j \in E} \rho_{j}^{2},
$$

where $T(E)$ is defined in (2.7).

End of the proof of Theorem 1.1. We assume that $h=1$ and that $\rho_{j}=\delta_{j} \leq 1$ for every $j \leq n$. According to $(2.10)$, we have

$$
\left\|O p^{W e y l}(F)\right\|_{\mathcal{L}\left(L^{2}\left(\mathbb{R}^{n}\right)\right)} \leq \sum_{E \subseteq\{1, \ldots, n\}}\left\|O p^{h y b, E}(T(E) F)\right\|_{\mathcal{L}\left(L^{2}\left(\mathbb{R}^{n}\right)\right)} .
$$

By Lemma 2.1:

$$
\left\|O p^{W e y l}(F)\right\|_{\mathcal{L}\left(L^{2}\left(\mathbb{R}^{n}\right)\right)} \leq \sum_{E \subseteq\{1, \ldots, n\}}\left(\frac{9 \pi}{2}\right)^{|E|} \sum_{(\alpha, \beta) \in I_{2}(E)}\left\|\partial_{x}^{\alpha} \partial_{\xi}^{\beta} T(E) F\right\|_{L^{\infty}\left(\mathbb{R}^{2 n}\right)}
$$


With the same hypotheses, Lemma 2.2 shows that

$$
\left\|O p^{W e y l}(F)\right\|_{\mathcal{L}\left(L^{2}\left(\mathbb{R}^{n}\right)\right)} \leq M \sum_{E \subseteq\{1, \ldots, n\}}\left(\frac{9 \pi}{2} \times 18\right)^{|E|} \prod_{j \in E} \rho_{j}^{2} .
$$

It follows easily that

$$
\left\|O p^{W e y l}(F)\right\|_{\mathcal{L}\left(L^{2}\left(\mathbb{R}^{n}\right)\right)} \leq M \prod_{j \leq n}\left(1+81 \pi \rho_{j}^{2}\right) .
$$

The theorem is proved in the case when $h=1$ and $\rho_{j}=\delta_{j} \leq 1$ for all $j \leq n$. In the general case, the announced result follows similarly.

\section{Proof of Lemma 2.1}

We shall use the results of Unterberger [21,22] concerning the upper bound of $\left\langle A \Psi_{X}, \Psi_{Y}\right\rangle$, where $A$ is a pseudodifferential operator and the $\Psi_{X}$ are the coherent states defined by (2.1). We first recall the integral expression of this scalar product and give an analogous statement for hybrid operators.

Proposition 3.1. Let $F$ be a function defined on $\mathbb{R}^{2 n}$ and satisfying hypothesis (H). Then we get, for every $X$ and $Y$ in $\mathbb{R}^{2 n}$,

$$
\left\langle O p^{W e y l}(F) \Psi_{X}, \Psi_{Y}\right\rangle=\pi^{-n} \int_{\mathbb{R}^{2 n}} F(Z) \Phi_{n}(X, Y, Z) d Z
$$

with

$$
\Phi_{n}(X, Y, Z)=e^{-\left|Z-\frac{X+Y}{2}\right|^{2}-i \sigma(Z, X-Y)-\frac{i}{2} \sigma(X, Y)},
$$

where the symplectic form $\sigma$ is given by $\sigma(X, Y)=y \cdot \xi-x \cdot \eta$ for all $X=(x, \xi)$ and $Y=(y, \eta)$ in $\mathbb{R}^{2 n}$.

Proof. For all functions $f$ and $g$ belonging to the Schwartz space $\mathcal{S}\left(\mathbb{R}^{n}\right)$, one defines the Wigner function $H(f, g, Z)\left(Z \in \mathbb{R}^{2 n}\right)$ by

$$
H(f, g, Z)=\int_{\mathbb{R}^{n}} e^{-i t \cdot \zeta} f\left(z+\frac{t}{2}\right) \overline{g\left(z-\frac{t}{2}\right)} d t, \quad Z=(z, \zeta) \in \mathbb{R}^{2 n}
$$

(cf. Unterberger [22, or Lerner [19], sections 2.1.1 and 2.1.2, or Combescure and Robert 4], section 2.2). The following equality is proved in [22] or [19] or [4, for all $f$ and $g$ in $\mathcal{S}\left(\mathbb{R}^{n}\right)$ and every Borel function $F$ which is bounded on $\mathbb{R}^{2 n}$ :

$$
\left\langle O p^{W e y l}(F) f, g\right\rangle=(2 \pi)^{-n} \int_{\mathbb{R}^{2 n}} F(Z) H(f, g, Z) d Z .
$$

An explicit computation using the coherent spaces $\Psi_{X}$ defined by (2.1) shows that

$$
H\left(\Psi_{X}, \Psi_{Y}, Z\right)=2^{n} \Phi_{n}(X, Y, Z),
$$

which implies (3.1).

Let $n^{\prime}<n$ and $n^{\prime \prime}=n-n^{\prime}$. We denote by $X=\left(X^{\prime}, X^{\prime \prime}\right)$ the variable in $\mathbb{R}^{2 n}$, with $X^{\prime}=\left(X_{1}, \ldots, X_{n^{\prime}}\right)$ and $X^{\prime \prime}=\left(X_{n^{\prime}+1}, \ldots, X_{n}\right)$. Set

$$
\Delta^{\prime \prime}=\sum_{j=n^{\prime}+1}^{n}\left(\partial_{x_{j}}^{2}+\partial_{\xi_{j}}^{2}\right) \text {. }
$$


Proposition 3.2. For all $f$ and $g$ in $\mathcal{S}\left(\mathbb{R}^{n}\right)$, we have

$$
\left\langle O p^{W e y l}\left(e^{\frac{1}{4} \Delta^{\prime \prime}} F\right) f, g\right\rangle
$$

$$
=C\left(n^{\prime}, n^{\prime \prime}\right) \int_{\mathbb{R}^{6 n^{\prime}+2 n^{\prime \prime}}} F\left(Z^{\prime}, T^{\prime \prime}\right) \Phi_{n^{\prime}}\left(X^{\prime}, Y^{\prime}, Z^{\prime}\right)\left\langle f, \Psi_{X^{\prime}, T^{\prime \prime}}\right\rangle\left\langle\Psi_{Y^{\prime}, T^{\prime \prime}}, g\right\rangle d X^{\prime} d Y^{\prime} d Z^{\prime} d T^{\prime \prime},
$$

where $C\left(n^{\prime}, n^{\prime \prime}\right)=2^{n^{\prime}}(2 \pi)^{-3 n^{\prime}-n^{\prime \prime}}, \Phi_{n^{\prime}}$ being the function defined by (3.2) with $n^{\prime}, X^{\prime}$ instead of $n, X$.

Proof. Proposition 3.1 and the integral expression for the heat operator $e^{\frac{1}{4} \Delta^{\prime \prime}}$ give (3.7)

$$
\left\langle O p^{W e y l}\left(e^{\frac{1}{4} \Delta^{\prime \prime}} F\right) \Psi_{X}, \Psi_{Y}\right\rangle=\pi^{-n-n^{\prime \prime}} \int_{\mathbb{R}^{2\left(n+n^{\prime \prime}\right)}} e^{-\left|Z^{\prime \prime}-T^{\prime \prime}\right|^{2}} F\left(Z^{\prime}, T^{\prime \prime}\right) \Phi_{n}(X, Y, Z) d Z d T^{\prime \prime} .
$$

An explicit computation yields

$$
\pi^{-n^{\prime \prime}} \int_{\mathbb{R}^{2 n^{\prime \prime}}} e^{-\left|Z^{\prime \prime}-T^{\prime \prime}\right|^{2}} \Phi_{n^{\prime \prime}}\left(X^{\prime \prime}, Y^{\prime \prime}, Z^{\prime \prime}\right) d Z^{\prime \prime}=2^{-n^{\prime \prime}}\left\langle\Psi_{X^{\prime \prime}}, \Psi_{T^{\prime \prime}}\right\rangle_{n^{\prime \prime}}\left\langle\Psi_{T^{\prime \prime}}, \Psi_{Y^{\prime \prime}}\right\rangle_{n^{\prime \prime}}
$$

where $\langle\cdot, \cdot\rangle_{n^{\prime \prime}}$ is the scalar product of $L^{2}\left(\mathbb{R}^{n^{\prime \prime}}\right)$. Combining (3.7) with this equality one sees that

$$
\left\langle O p^{W e y l}\left(e^{\frac{1}{4} \Delta^{\prime \prime}} F\right) \Psi_{X}, \Psi_{Y}\right\rangle
$$

$$
=2^{-n^{\prime \prime}} \pi^{-n} \int_{\mathbb{R}^{2 n}} F\left(Z^{\prime}, T^{\prime \prime}\right) \Phi_{n^{\prime}}\left(X^{\prime}, Y^{\prime}, Z^{\prime}\right)\left\langle\Psi_{X^{\prime \prime}}, \Psi_{T^{\prime \prime}}\right\rangle_{n^{\prime \prime}}\left\langle\Psi_{T^{\prime \prime}}, \Psi_{Y^{\prime \prime}}\right\rangle_{n^{\prime \prime}} d Z^{\prime} d T^{\prime \prime} .
$$

For all $f$ and $g$ in $\mathcal{S}\left(\mathbb{R}^{n}\right)$, one gets, applying (2.2) twice

$$
\begin{gathered}
\left\langle O p^{W e y l}\left(e^{\frac{1}{4} \Delta^{\prime \prime}} F\right) f, g\right\rangle \\
=(2 \pi)^{-2 n} \int_{\mathbb{R}^{4 n}}\left\langle f, \Psi_{X}\right\rangle\left\langle O p^{W e y l}\left(e^{\frac{1}{4} \Delta^{\prime \prime}} F\right) \Psi_{X}, \Psi_{Y}\right\rangle\left\langle\Psi_{Y}, g\right\rangle d X d Y .
\end{gathered}
$$

One then applies (3.8) and the following result, deduced from (2.2) in dimension $n^{\prime \prime}$ :

$$
(2 \pi)^{-n^{\prime \prime}} \int_{\mathbb{R}^{2 n^{\prime \prime}}}\left\langle f, \Psi_{X}\right\rangle\left\langle\Psi_{X^{\prime \prime}}, \Psi_{T^{\prime \prime}}\right\rangle_{n^{\prime \prime}} d X^{\prime \prime}=\left\langle f, \Psi_{X^{\prime}, T^{\prime \prime}}\right\rangle .
$$

Formula (3.6) follows from that and from an analogous result about $\left\langle\Psi_{Y}, g\right\rangle$.

For every $X^{\prime}=\left(x^{\prime}, \xi^{\prime}\right)$ in $\mathbb{R}^{2 n^{\prime}}$, set

$$
K_{n^{\prime}}\left(X^{\prime}\right)=\prod_{j=1}^{n^{\prime}}\left(1+x_{j}^{2}\right)\left(1+\xi_{j}^{2}\right) .
$$

Lemma 3.3. For every function $G$ satisfying hypothesis $(H)$, for every $X^{\prime}$ and $Y^{\prime}$ in $\mathbb{R}^{2 n^{\prime}}$ and $Z^{\prime \prime}$ in $\mathbb{R}^{2 n^{\prime \prime}}$,

$$
\begin{gathered}
K_{n^{\prime}}\left(X^{\prime}-Y^{\prime}\right)\left|\pi^{-n^{\prime}} \int_{\mathbb{R}^{2 n^{\prime}}} F\left(Z^{\prime}, Z^{\prime \prime}\right) \Phi_{n^{\prime}}\left(X^{\prime}, Y^{\prime}, Z^{\prime}\right) d Z^{\prime}\right| \leq 9^{n^{\prime}} N_{n^{\prime}}(F), \\
N_{n^{\prime}}(F)=\sum_{(\alpha, \beta) \in I_{2}\left(n^{\prime}\right)}\left\|\partial_{x}^{\alpha} \partial_{\xi}^{\beta} F\right\|_{L^{\infty}\left(\mathbb{R}^{2 n}\right)},
\end{gathered}
$$

where $I_{2}\left(n^{\prime}\right)$ is the set of multi-indices $\alpha$ such that $\alpha_{j} \leq 2$ and $\beta_{j} \leq 2$ for all $j \leq n$, and $\alpha_{j}=\beta_{j}=0$ for $j>n^{\prime}$. 
Proof. Let $I\left(X^{\prime}, Y^{\prime}, Z^{\prime \prime}\right)$ be the left side of (3.11). Integration by parts shows that for all $X$ and $Y$ in $\mathbb{R}^{2 n^{\prime}}$,

$$
I\left(X^{\prime}, Y^{\prime}, Z^{\prime \prime}\right) \leq \pi^{-n^{\prime}} \int_{\mathbb{R}^{2 n^{\prime}}}\left|(L F)\left(Z^{\prime}+\frac{X^{\prime}+Y^{\prime}}{2}, Z^{\prime \prime}\right)\right| e^{-\left|Z^{\prime}\right|^{2}} d Z^{\prime},
$$

where $L$ is the differential operator defined by

$$
\begin{aligned}
& L=\prod_{j \leq n^{\prime}} L_{z_{j}} L_{\zeta_{j}}, \quad L_{z_{j}}=\sum_{k=0}^{2} a_{k}\left(z_{j}\right) \partial_{z_{j}}^{k}, \\
& a_{0}(z)=3-4 z^{2}, \quad a_{1}(z)=4 z, \quad a_{2}(z)=-1 .
\end{aligned}
$$

We get as a consequence that

$$
I\left(X^{\prime}, Y^{\prime}, Z^{\prime \prime}\right) \leq \sum_{(\alpha, \beta) \in I_{2}\left(n^{\prime}\right)}\left\|\partial_{x}^{\alpha} \partial_{\xi}^{\beta} F\right\|_{L^{\infty}\left(\mathbb{R}^{2 n}\right)} \prod_{j \leq n^{\prime}} C_{\alpha_{j}} C_{\beta_{j}}
$$

with

$$
C_{k}=\pi^{-1 / 2} \int_{\mathbb{R}}\left|a_{k}(z)\right| e^{-z^{2}} d z, \quad \quad k=0,1,2 .
$$

The formula (3.11) then follows from the fact that $\max \left(C_{0}, C_{1}, C_{2}\right) \leq 3$.

End of the proof of Lemma 2.1. The subset $E$ may be any subset of $\{1, \ldots, n\}$, but we can assume in the proof that $E=\left\{1, \ldots, n^{\prime}\right\}$ with $0 \leq n^{\prime} \leq n$. In this case, we use the above notation, and we set $x=\left(x^{\prime}, x^{\prime \prime}\right)$ with $x^{\prime}=\left(x_{1}, \ldots, x_{n^{\prime}}\right)$ and $x^{\prime \prime}=\left(x_{n^{\prime}+1}, \ldots, x_{n}\right)$, etc.

Using (3.6) and Lemma 3.3 to obtain an upper bound on the right side, one gets

$$
\begin{gathered}
\left|\left\langle O p^{W e y l}\left(e^{\frac{1}{4} \Delta^{\prime \prime}} F\right) f, g\right\rangle\right| \\
\leq 9^{n^{\prime}}(2 \pi)^{-2 n^{\prime}-n^{\prime \prime}} N_{n^{\prime}}(F) \int K_{n^{\prime}}\left(X^{\prime}-Y^{\prime}\right)^{-1}\left|\left\langle f, \Psi_{X^{\prime}, T^{\prime \prime}}\right\rangle\left\langle\Psi_{Y^{\prime}, T^{\prime \prime}}, g\right\rangle\right| d X^{\prime} d Y^{\prime} d T^{\prime \prime} .
\end{gathered}
$$

According to Schur's Lemma, this is smaller than

$$
\begin{gathered}
\cdots \leq 9^{n^{\prime}}(2 \pi)^{-2 n^{\prime}-n^{\prime \prime}} N_{n^{\prime}}(F)\left\|K_{n^{\prime}}^{-1}\right\|_{L^{1}\left(\mathbb{R}^{2 n^{\prime}}\right)} \\
\ldots\left[\int\left|\left\langle f, \Psi_{X^{\prime}, T^{\prime \prime}}\right\rangle\right|^{2} d X^{\prime} d T^{\prime \prime}\right]^{1 / 2}\left[\int\left|\left\langle\Psi_{Y^{\prime}, T^{\prime \prime}}, g\right\rangle\right|^{2} d Y^{\prime} d T^{\prime \prime}\right]^{1 / 2} .
\end{gathered}
$$

Using (2.2), one shows that $\left|\left\langle O p^{W e y l}\left(e^{\frac{1}{4} \Delta^{\prime \prime}} F\right) f, g\right\rangle\right|$ is smaller than

$$
\leq 9^{n^{\prime}}(2 \pi)^{-n^{\prime}} N_{n^{\prime}}(F)\left\|K_{n^{\prime}}^{-1}\right\|_{L^{1}\left(\mathbb{R}^{2 n^{\prime}}\right)}\|f\|_{L^{2}\left(\mathbb{R}^{n}\right)}\|g\|_{L^{2}\left(\mathbb{R}^{n}\right)} .
$$

Since $\left\|K_{n^{\prime}}^{-1}\right\|_{L^{1}\left(\mathbb{R}^{2 n^{\prime}}\right)}=\pi^{2 n^{\prime}}$, the former inequalities imply that

$$
\left|\left\langle O p^{W e y l}\left(e^{\frac{1}{4} \Delta^{\prime \prime}} F\right) f, g\right\rangle\right| \leq\left(\frac{9 \pi}{2}\right)^{n^{\prime}} N_{n^{\prime}}(F)\|f\|_{L^{2}\left(\mathbb{R}^{n}\right)}\|g\|_{L^{2}\left(\mathbb{R}^{n}\right)} .
$$

Lemma 2.1 holds if $E=\left\{1, \ldots, n^{\prime}\right\}$, a case which we are brought back to by a suitable permutation. 


\section{Proof of Lemma 2.2}

Let $\Delta_{j}$ be the operator defined by $(2.6)$ and

$$
A_{j}=I-e^{\frac{1}{4} \Delta_{j}} .
$$

Lemma 4.1. One can write

$$
A_{j}=B_{j} \partial_{x_{j}}+C_{j} \partial_{\xi_{j}}=D_{j} \Delta_{j},
$$

where the operators $B_{j}, C_{j}$ and $D_{j}$ are bounded in the space $C_{b}$ of continuous bounded functions on $\mathbb{R}^{2 n}$. More precisely,

$$
\begin{array}{cr}
\left\|A_{j}\right\|_{\mathcal{L}\left(C_{b}\right)} \leq 2, & \left\|B_{j}\right\|_{\mathcal{L}\left(C_{b}\right)} \leq \pi^{-1 / 2} \\
\left\|C_{j}\right\|_{\mathcal{L}\left(C_{b}\right)} \leq \pi^{-1 / 2}, & \left\|D_{j}\right\|_{\mathcal{L}\left(C_{b}\right)} \leq 1 / 4 .
\end{array}
$$

Proof. The first inequality in (4.3) is standard. The expression of the heat operator allows us to write the first equality in (4.2) with

$$
\begin{aligned}
& \left(B_{j} \varphi\right)(x, \xi)=-\pi^{-1} \int_{\mathbb{R}^{2} \times[0,1]} e^{-\left(u^{2}+v^{2}\right)} u \varphi\left(x+\theta u e_{j}, \xi+\theta v e_{j}\right) d u d v d \theta, \\
& \left(C_{j} \varphi\right)(x, \xi)=-\pi^{-1} \int_{\mathbb{R}^{2} \times[0,1]} e^{-\left(u^{2}+v^{2}\right)} v \varphi\left(x+\theta u e_{j}, \xi+\theta v e_{j}\right) d u d v d \theta .
\end{aligned}
$$

We deduce the bounds on the norms of $C_{j}$ and $D_{j}$ in (4.3) from these inequalities. The last inequality (4.2) and the bound on $D_{j}$ in (4.3) follow by integrating by parts in (4.4) and (4.5).

End of the proof of Lemma 2.2. For every multi-index $(\alpha, \beta)$ in $I_{2}(E)$, one can rewrite the operator $\partial_{x}^{\alpha} \partial_{\xi}^{\beta} T(E)$ as follows:

$$
\partial_{x}^{\alpha} \partial_{\xi}^{\beta} T(E)=\prod_{j \in E} U_{j} \partial_{x}^{\alpha_{j}} \partial_{\xi}^{\beta_{j}}
$$

with

$$
U_{j}=\left\{\begin{array}{llc}
A_{j} & \text { if } & \alpha_{j}+\beta_{j} \geq 2 \\
B_{j} \partial_{x_{j}}+C_{j} \partial_{\xi_{j}} & \text { if } & \alpha_{j}+\beta_{j}=1 \\
D_{j} \Delta_{j} & \text { if } & \alpha_{j}+\beta_{j}=0
\end{array}\right.
$$

According to the bounds on the norms of the operators $A_{j}$ to $D_{j}$ given by Lemma 4.1, if $F$ satisfies hypothesis $(\mathrm{H})$ with $\rho_{j}=\delta_{j} \leq 1$, one has

$$
\left\|\partial_{x}^{\alpha} \partial_{\xi}^{\beta} T(E) F\right\|_{L^{\infty}\left(\mathbb{R}^{2 n}\right)} \leq M 2^{|E|} \prod_{j \in E} \rho_{j}^{2} .
$$

Since $I_{2}(E)$ contains exactly $9^{|E|}$ elements, this achieves the proof of Lemma 2.2.

\section{ACKNOWLEDGEMENTS}

The authors express their thanks to the referee for helpful suggestions which allowed them, in particular, to gain on the number of derivatives and to simplify the proofs. 


\section{REFERENCES}

[1] L. Amour, L. Jager, and J. Nourrigat, Bounded Weyl pseudodifferential operators in Fock space, preprint, arXiv:1209.2852, Sept. 2012.

[2] Alberto-P. Calderón and Rémi Vaillancourt, A class of bounded pseudo-differential operators, Proc. Nat. Acad. Sci. U.S.A. 69 (1972), 1185-1187. MR0298480 (45 \#7532)

[3] Ronald R. Coifman and Yves Meyer, Au delà des opérateurs pseudo-différentiels (French), Astérisque, vol. 57, Société Mathématique de France, Paris, 1978. With an English summary. MR.518170 (81b:47061)

[4] Monique Combescure and Didier Robert, Coherent states and applications in mathematical physics, Theoretical and Mathematical Physics, Springer, Dordrecht, 2012. MR2952171

[5] H. O. Cordes, On compactness of commutators of multiplications and convolutions, and boundedness of pseudodifferential operators, J. Funct. Anal. 18 (1975), 115-131. MR0377599 $(51 \# 13770)$

[6] Gerald B. Folland, Harmonic analysis in phase space, Annals of Mathematics Studies, vol. 122, Princeton University Press, Princeton, NJ, 1989. MR983366 (92k:22017)

[7] L. Hörmander, The analysis of linear partial differential operators, Volume III, Springer, 1985. MR0781536 (87d:35002a)

[8] I. L. Hwang, The $L^{2}$ boundedness of pseudo-differential operators, Trans. Amer. Math. Soc. 302 (1987), no. 1, 55-76. MR0887496 (88e:47096)

[9] B. Lascar, Noyaux d'une classe d'opérateurs pseudo-différentiels sur l'espace de Fock, et applications. Séminaire Paul Krée, 3e année (1976-77), Equations aux dérivées partielles en dimension infinie, Exp. No. 6, 43 pp. Lecture Notes in Math., 650, Springer, Berlin, 1978. MR0504779 (81e:47040)

[10] Bernard Lascar, Équations aux dérivées partielles en dimension infinie (French), Vector space measures and applications (Proc. Conf., Univ. Dublin, Dublin, 1977), I, Lecture Notes in Math., vol. 644, Springer, Berlin, 1978, pp. 286-313. MR502411(81d:58021)

[11] Bernard Lascar, Opérateurs pseudo-différentiels en dimension infinie. Étude de l'hypoellipticité de la résolubilité dans des classes de fonctions holderiennes et de distributions pour des opérateurs pseudo-différentiels elliptiques (French), J. Analyse Math. 33 (1978), 39-104, DOI 10.1007/BF02790168. MR516042(81a:35097)

[12] B. Lascar, Une classe d'opérateurs elliptiques du second ordre sur un espace de Hilbert (French), J. Funct. Anal. 35 (1980), no. 3, 316-343, DOI 10.1016/0022-1236(80)90086-5. MR.563559(81i:47052)

[13] Bernard Lascar, Opérateurs pseudo-différentiels en dimension infinie. Applications (French, with English summary), C. R. Acad. Sci. Paris Sér. A-B 284 (1977), no. 13, A767-A769. MR0473820 (57 \#13481)

[14] B. Lascar, Méthodes $L^{2}$ pour des équations aux dérivées partielles dépendant d'une infinité de variables (French), Séminaire Goulaouic-Schwartz (1975/1976) Équations aux dérivées partielles et analyse fonctionnelle, Exp. No. 5, Centre Math., École Polytech., Palaiseau, 1976, pp. 11. MR0464964 (57 \#4883)

[15] B. Lascar, Propriétés locales d'espaces de type Sobolev en dimension infinie (French), Comm. Partial Differential Equations 1 (1976), no. 6, 561-584. MR0415316 (54 \#3405)

[16] B. Lascar, Propriétés locales d'espaces de type Sobolev en dimension infinie. Séminaire Paul Krée, 1re année (1974-75), Equations aux dérivées partielles en dimension infinie, Exp. No. 11, 16 pp. MR 0482203

[17] B. Lascar, Opérateurs pseudo-différentiels d'une infinité de variables, d'après M. I. Visik (French), Séminaire Pierre Lelong (Analyse), Année 1973-1974, Springer, Berlin, 1975, pp. 83-90. Lecture Notes in Math., Vol. 474. MR0420265 (54 \#8279)

[18] Bernard Lascar, Propriétés d'espaces de Sobolev en dimension infinie (French, with English summary), C. R. Acad. Sci. Paris Sér. A-B 280 (1975), no. 23, Ai, A1587-A1590. MR0390769 (52\#11592)

[19] Nicolas Lerner, Metrics on the phase space and non-selfadjoint pseudo-differential operators, Pseudo-Differential Operators. Theory and Applications, vol. 3, Birkhäuser Verlag, Basel, 2010. MR2599384(2011b:35002)

[20] Didier Robert, Autour de l'approximation semi-classique (French), Progress in Mathematics, vol. 68, Birkhäuser Boston Inc., Boston, MA, 1987. MR897108 (89g:81016) 
[21] André Unterberger, Oscillateur harmonique et opérateurs pseudo-différentiels (French, with English summary), Ann. Inst. Fourier (Grenoble) 29 (1979), no. 3, xi, 201-221. MR552965 (81m:58077)

[22] A. Unterberger, Les opérateurs métadifférentiels (French), (Proc. Internat. Colloq., Centre Phys., Les Houches, 1979), Lecture Notes in Phys., vol. 126, Springer, Berlin, 1980, pp. 205241. MR:579751 (82j:35142)

Laboratoire de Mathématiques, EA 4535, FR CNRS-3399, Université de Reims Champagne-Ardenne, 51687 Reims, France

E-mail address: laurent.amour@univ-reims.fr

Laboratoire de Mathématiques, EA 4535, FR CNRS-3399, Université de Reims Champagne-Ardenne, 51687 Reims, France

E-mail address: lisette.jager@univ-reims.fr

Laboratoire de Mathématiques, EA 4535, FR CNRS-3399, Université de Reims Champagne-Ardenne, 51687 Reims, France

E-mail address: jean.nourrigat@univ-reims.fr 